\title{
EVALUACIÓN DEL EFECTO ANTIMICROBIANO DE UNA MEZCLA DE PROBIÓTICOS FRENTE A CRONOBACTER SAKAZAKII Y SALMONELLA ENTERICA EN PREPARADOS RECONSTITUIDOS PARA LACTANTES
}

Evaluation of the antimicrobial effect of a probiotic mixture against Cronobacter sakazakii and Salmonella enterica in infant formulas

\section{Candel-Pérez, C.; García-Iborra R.; García-Alonso, J.; Martínez-Graciá, C.*}

Departamento de Tecnología de los Alimentos, Nutrición y Bromatología, Facultad de Veterinaria, Campus de Excelencia Regional Mare Nostrum, Universidad de Murcia, España

*Autor de correspondencia: Carmen Martínez Graciá. Universidad de Murcia, Departamento de Nutrición y Bromatología, Facultad de Veterinaria, Campus Espinardo, Murcia 30100, España. E-mail: mamen@um.es

Historial del artículo:

Enviado: 12 de diciembre, 2019

Aceptado: 5 de marzo, 2020

\section{RESUMEN}

Cronobacter sakazakii y Salmonella enterica son considerados patógenos capaces de causar sepsis y meningitis en lactantes. Se han descrito casos de infección por estos patógenos asociados al consumo de preparados para lactantes contaminados. La inclusión de probióticos en los preparados, además de su papel en la modulación de la microbiota intestinal, podría ser útil para contrarrestar la contaminación de microorganismos patógenos de estos productos una vez reconstituidos y, por lo tanto, reducir el riesgo de infección. El objetivo de esta investigación fue evaluar el efecto antimicrobiano de una mezcla de probióticos (Lactobacillus rhamnosus y Bifidobacterium infantis) frente a Cronobacter sakazakii y Salmonella enterica en preparados reconstituidos para lactantes. Las muestras (con o sin probióticos) se inocularon con C. sakazakii o $S$. enterica y se incubaron a 30 o $40^{\circ} \mathrm{C}$ durante 72 horas. Los resultados muestran que la presencia de probióticos en los preparados lácteos no tiene un marcado efecto en el crecimiento de los patógenos. Sin embargo sí se ve potenciado su efecto inhibitorio frente a C. sakazakii por la interacción entre la temperatura de incubación y los probióticos. Los probióticos empleados fueron viables durante todo el período de incubación, por lo que el agotamiento de los nutrientes se descartó como posible mecanismo de inhibición. La disminución del pH en los preparados lácteos inoculados no explica de manera 
satisfactoria un comportamiento tan diferente entre ambos patógenos. Por lo tanto, se sugiere como posible mecanismo inhibidor la liberación de bacteriocinas específicas con acción contra C . sakazakii pero sin efecto contra Salmonella. Se deben realizar estudios adicionales para probar esta hipótesis.

Palabras clave: Preparados para lactantes, Cronobacter sakazakii, Salmonella enterica, bacteriocinas, Bifidobacterium infantis, Lactobacillus rhamnosus

\begin{abstract}
Cronobacter sakazakii and Salmonella enterica have been implicated in outbreaks causing sepsis and meningitis in infants. Several infection cases have been associated with the consumption of contaminated powdered infant formulae. Besides its role in modulating gut microbiota, the inclusion of probiotics in infant formulae may be useful to counteract pathogen contamination in these products and thus to reduce the risk of infection. The aim of this research was to evaluate the antimicrobial effect of a mixture of the probiotics Lactobacillus rhamnosus and Bifidobacterium infantis against Cronobacter sakazakii and Salmonella enterica in reconstituted follow-on formulae. For that, infant formulae (with or without probiotics) were inoculated with either $C$. sakazakii or S. enterica and incubated at 30 or $40^{\circ} \mathrm{C}$ for 72 hours. Overall, the results showed the presence of probiotics inhibiting the growth of $C$. sakazakii together with incubation temperature (interaction between both factors). Probiotics were viable throughout the incubation period so that nutrient exhaustion could be ruled out as the mechanism of $C$. sakazakii inhibition. Thus, the acidification of media observed during the study was hypothesized to be responsible for pathogen growth inhibition. However, since in the case of formulae inoculated with Salmonella the behavior of probiotics and $\mathrm{pH}$ values was similar to C. sakazakii, the decrease of $\mathrm{pH}$ does not satisfactorily explain such a different behavior. So, it is suggested that the production of specific bacteriocins by probiotics against $C$. sakazakii but not against Salmonellla could be the underlying inhibitory mechanism. Further studies should be carried out to test this hypothesis.
\end{abstract}

Keywords: Follow-on infant formula, Cronobacter sakazakii, Salmonella enterica, bacteriocinas, Bifidobacterium infantis, Lactobacillus rhamnosus

\section{INTRODUCCIÓN}

Los preparados para lactantes de continuación son alimentos destinados a lactantes de entre 5 meses y 3 años que se introducen en la alimentación complementaria y constituyen el principal elemento líquido en una dieta progresivamente diversificada (Comisión Europea, 2016). Con objeto de simular algunos de los aspectos beneficiosos de la leche materna, existen fórmulas de continuación suplementadas con simbióticos -una mezcla de probióticos y prebióticos- capaces de beneficiar al huésped, al mejorar la supervivencia y la implantación de los probióticos en el tracto gastrointestinal (Braegger et al., 2011). La mayoría de los probióticos empleados son bacterias ácido lácticas (BAL) pertenecientes a los géneros Bifidobacterium y Lactobacillus, que presentan excelen- tes propiedades preventivas frente a diversas patologías (Kopp-Hoolihan, 2001). Varias evidencias demuestran que la suplementación de preparados para lactantes con una mezcla de diferentes prebióticos induce una modificación significativa en la composición de la microbiota intestinal de recién nacidos y prematuros, aumentando el número de bacterias ácido lácticas en el colon (Markowiak and Ślizewska, 2017).

Las fórmulas en polvo no son un producto estéril y pueden estar intrínsecamente contaminadas con microorganismos patógenos. Expertos de la Organización de las Naciones Unidas para la Agricultura y la Alimentación y la Organización Mundial de la Salud concluyeron que los microorganismos de mayor preocupación en los preparados para lactantes en polvo son Cronobacter sakazakii y Salmonella entérica (FAO/WHO, 2006), ya que se ha demostrado 
de manera convincente que estos alimentos contaminadas con dichos patógenos son fuente y vehículo de infección para los lactantes. De hecho, C. sakazakii y Salmonella enterica, y otros bacilos Gram negativos pertenecientes a la familia Enterobacteriaceae, se han visto implicados en casos de meningitis, encefalitis, septicemia y enterocolitis necrotizante en lactantes (Usera et al., 1996; Drudy et al., 2006; Park and Soo-Hwan, 2007; Simón et al., 2010; Salimiyan et al., 2019).

Las posibles rutas por las que $C$. sakazakii y Salmonella pueden estar presentes en los preparados y llegar al lactante, puede ser a través de los ingredientes utilizados para prepararlas o por contaminación cruzada durante su reconstitución o almacenamiento. Los principales factores contribuyentes al riesgo de infección son la inadecuada temperatura utilizada durante la reconstitución del preparado en polvo $\left(<70{ }^{\circ} \mathrm{C}\right)$ y el prolongado período de conservación de los biberones a temperaturas superiores a la mínima de crecimiento $\left(5,5^{\circ} \mathrm{C}\right)$ de los patógenos, antes de su utilización (EFSA, 2004; WHO/ FAO, 2007).

La termo-resistencia de C. sakazakii y Salmonella, combinada con la capacidad de persistir en ambientes secos, permite su supervivencia y crecimiento en estos alimentos (Park and SooHwan, 2007; Dancer, 2009, Bai et al., 2019; Kingsbury et al., 2019). Además, C. sakazakii y Salmonella son capaces de multiplicarse rápidamente en el producto reconstituido a temperatura ambiente, incluso si se almacenan a más de $5{ }^{\circ} \mathrm{C}$ durante el tiempo suficiente $(\mathrm{FAO} /$ WHO, 2006). Por tanto, un nivel bajo de contaminación podría verse incrementado en función de las condiciones higiénicas adoptadas durante la reconstitución, así como por la duración y la temperatura de almacenamiento (Iversen et al., 2004; Hurrell et al., 2009; Al-Nabulsi et al., 2011; Ghassem et al., 2011).

Aunque la capacidad de C. sakazakii y Salmonella para crecer en preparados para lactantes en polvo y reconstituidos ha sido estudia- da por diversos autores (Iversen et al., 2004; Kandhai et al., 2006; Lenati et al., 2008), su comportamiento en preparados con probióticos, y a diferentes temperaturas de conservación tras su reconstitución, no se ha estudiado completamente. El objetivo de este estudio fue evaluar el efecto antimicrobiano de una mezcla de probióticos (Lactobacillus rhamnosus y Bifidobacterium infantis) frente a Cronobacter sakazakii y Salmonellla enterica en preparados de continuación reconstituidos para lactantes.

\section{MATERIAL Y MÉTODOS}

\subsection{Preparados de continuación para lactantes}

Se prepararon cuatro muestras reconstituidas de $170 \mathrm{~mL}$, en botellas de vidrio estériles, suplementadas con probióticos a partir de un preparado comercial para lactantes compuesto por una mezcla de $2.0 \times 10^{9} \mathrm{UFC} / 100 \mathrm{~g}$ Lactobacillus rhamnosus y $2.7 \times 10^{8} \mathrm{UFC} / 100 \mathrm{~g}$ Bifidobacterium infantis. Como control, se prepararon otras cuatro muestras reconstituidas de $170 \mathrm{~mL}$ con el mismo preparado comercial de continuación sin probióticos (también disponible de forma comercial). Ambos tipos de muestras, adquiridas en una farmacia local, se prepararon de acuerdo con las instrucciones del fabricante (20 g por $150 \mathrm{ml}$ de agua) utilizando agua destilada estéril calentada a $40^{\circ} \mathrm{C}$ en un baño de agua.

\subsection{Medios de cultivo y microorganismos}

Se prepararon los siguientes medios de cultivo, de acuerdo con las instrucciones de los fabricantes: agua de peptona (BPW) (Oxoid, Reino Unido); agar Man Rogosa Sharpe (MRS) (Oxoid, Reino Unido) suplementado con 0,25\% de L-cisteína (Sigma-Aldrich, EE. UU.); agar VRBGA (Oxoid, Reino Unido); agar TSA (Oxoid, Reino Unido); solución salina tamponada con fosfato (PBS) pH 7.4 (Sigma-Aldrich, St. USA); caldo BHI (Scharlau, Scharlab, S.L., Italy). Se adquirieron los siguientes medios de 
cultivo listos para utilizar: agar E. sakazakii (ESIA) (AES Laboratoire), caldo selectivo de E. sakazakii (ESSB) (AES Laboratoire), Agar Salmonellla-Shigella (SS) (BioMérieux, Francia) y caldo selenito (Difco, España).

La cepa de Enterobacter sakazakii CECT 858, suministrada por la Colección Española de Cultivos Tipo (lote 05-10-2006), se reconstituyó en caldo ESSB para posteriormente aislarse en agar ESIA. Salmonella enterica serotipo D, fue cedida por el Laboratorio de Microbiología del hospital Virgen de la Arrixaca, aislándose en agar SS a partir de una muestra de heces.

\subsection{Diseño experimental}

Se realizaron cuatro ensayos para estudiar el crecimiento y la supervivencia de Salmonella y C. sakazakii a dos temperaturas de incubación diferentes. La Figura 1 muestra cómo los preparados reconstituidos para lactantes (con o sin probióticos) se inocularon con $C$. sakazakii o con Salmonella enterica, y se incubaron a 30 o $40^{\circ} \mathrm{C}$ durante $72 \mathrm{~h}$.

Los inóculos se prepararon transfiriendo una colonia bacteriana de C. sakazakii o Salmonella enterica a $2 \mathrm{~mL}$ de solución salina estéril. Esta suspensión se ajustó a la escala McFarland de 0,5 puntos con un nefelómetro $(600 \mathrm{~nm})$ (Densimat ${ }^{\circledR}$, BioMérieux, Francia), equivalente a una concentración de aproximadamente $10^{8}$ UFC / mL y se diluyó con agua de peptona estéril hasta obtener una concentración de $10^{5}$ $\mathrm{UFC} / \mathrm{mL}$. Las muestras reconstituidas $(170 \mathrm{~mL})$ se inocularon con 1,7 mL de esta suspensión. El nivel inicial de C. sakazakii y Salmonellla enterica en la preparación reconstituida fue de aproximadamente $10^{3} \mathrm{UFC} / \mathrm{mL}$.

Se tomaron alícuotas de cada muestra reconstituida a las $0,4,16,24,48$ y 72 horas de incubación a 30 y $40^{\circ} \mathrm{C}$. Una alícuota de $1 \mathrm{~mL}$ se empleó para realizar diluciones seriadas 1:10 en PBS hasta $10^{-9}$. De cada dilución se sembraron 0,1 mL en agar TSA, VRBG y MRS mediante la técnica de extensión en superficie. Las placas de TSA y VRBG se incubaron 24 horas a $37^{\circ} \mathrm{C}$, mientras que las placas de MRS se incubaron durante 48 horas a $37^{\circ} \mathrm{C}$ en una jarra hermética que contenía un sobre de anaerobiosis (Oxoid, Reino Unido). Transcurrida la incubación, se realizó visualmente el recuento bacteriano de enterobacterias en VRBG y de los probióticos en MRS. El agar TSA se empleó para descartar la contaminación extrínseca de las muestras reconstituidas durante el estudio. Además, una alícuota de $100 \mu \mathrm{L}$ de los preparados se sembró en ESIA o SS para confirmar el crecimiento de C. sakazakii o Salmonella, respectivamente, en el reconstituido (límite de detección de 10 UFC/ $\mathrm{mL})$. Para completar los ensayos, otra alícuota de las muestras $(10 \mathrm{~mL})$ se empleó para realizar una medida de $\mathrm{pH}$ (microPH 2000, Crison Instruments S.A., Barcelona, España). Todos los ensayos se realizaron por duplicado.

\subsection{Identificación bacteriana}

Las colonias azuladas en agar ESIA, susceptibles de ser C. sakazakii, y las colonias negras en SS, potencialmente de Salmonella spp., se identificaron por su perfil bioquímico con una galería API20E (bioMérieux Reino Unido), de acuerdo con las instrucciones del fabricante. Mediante la base de datos apiweb ${ }^{\mathrm{TM}}$ (versión 4.0) se identificaron las presuntas colonias de Cronobacter al nivel de especie como Enterobacter sakazakii con identificación "buenaexcelente" (98.4\%), y se identificó Salmonella con identificación "buena-excelente" (99.9\%). La confirmación serológica de Salmonella se llevó a cabo mediante aglutinación en portaobjetos con antisueros de agrupamiento de Salmonella D (Difco Salmonella O Antiserum Factor 9, Becton Dickinson, EE. UU.), de acuerdo con las instrucciones del fabricante.

La identificación fenotípica de los probióticos se basó en las características macroscópicas de las colonias en el medio MRS y por su morfología microscópica tras realizar la tinción de Gram. 


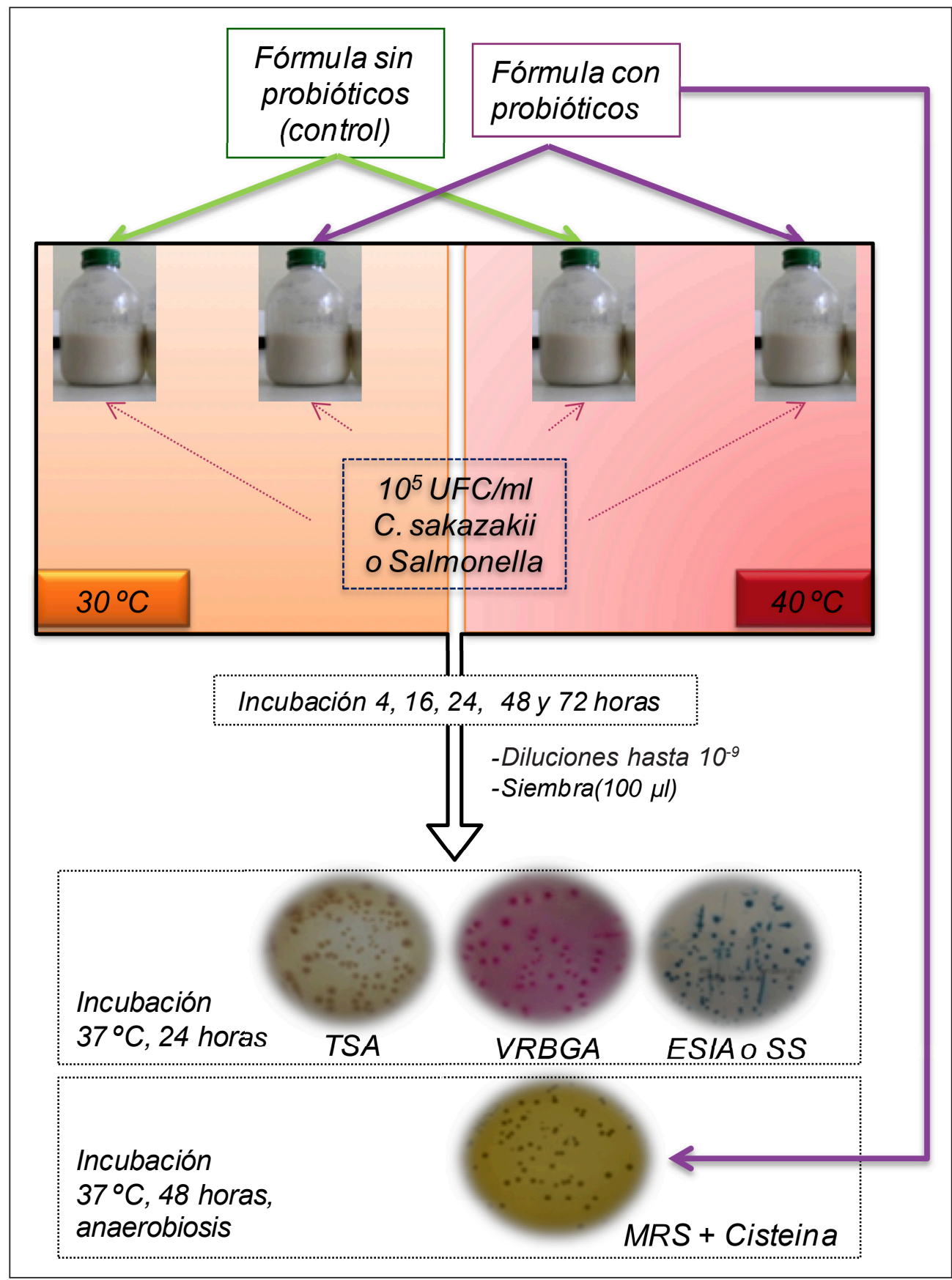

Figura 1. Diseño experimental: los preparados para lactantes (con o sin probióticos) se inocularon con $C$. sakazakii o con Salmonella enterica, y se incubaron a 30 o $40^{\circ} \mathrm{C}$ durante $72 \mathrm{~h}$ 


\subsection{Análisis estadístico}

Los datos se analizaron con el paquete estadístico SPSS (versión 15.0) para Windows. Para estudiar el efecto de la presencia de probióticos, el tiempo de incubación y la temperatura de incubación sobre el crecimiento de los patógenos, se realizó un análisis de varianza (ANOVA) utilizando el modelo lineal general (GLM), considerando $\mathrm{P}<0,05$ como el nivel de significancia estadística.

\section{RESULTADOS}

La Tabla 1 y 2 muestran los valores de $\mathrm{pH}$ y recuentos de $C$. sakazakii y probióticos obtenidos a partir de los preparados reconstituidos almacenados a 30 y $40{ }^{\circ} \mathrm{C}$ respectivamente. Los recuentos de $C$. sakazakii sufrieron variaciones en función a las condiciones de estudio. En las muestras con probióticos, tras la incubación a $30{ }^{\circ} \mathrm{C}$ durante 72 horas, la concentración de C. sakazakii fue de $3.3 \log \mathrm{UFC} / \mathrm{mL}(\mathrm{p}<0,05)$

Tabla 1. Valores de $\mathbf{p H}$ y recuentos de Cronobacter sakazakii y probióticos de los preparados reconstituidos para lactantes y almacenadas a $30{ }^{\circ} \mathrm{C}$

\begin{tabular}{llllll}
\hline $\begin{array}{l}\text { Tiempo de } \\
\text { incubación } \\
\text { (horas) }\end{array}$ & \multicolumn{3}{l}{ Preparados sin probióticos } & \multicolumn{3}{l}{ Preparados con probióticos } \\
\cline { 2 - 6 } & $\mathrm{pH}$ & $\begin{array}{l}\text { C. sakazakii } \\
(\log \mathrm{UFC} / \mathrm{mL})\end{array}$ & $\mathrm{pH}$ & $\begin{array}{l}\text { Probióticos(log } \\
\mathrm{UFC} / \mathrm{mL})\end{array}$ & $\begin{array}{l}\text { C. sakazakii } \\
\text { (log UFC/mL) }\end{array}$ \\
\hline 0 & 6.87 & 2.70 & 6.64 & 7.00 & 2.45 \\
4 & 6.79 & 4.00 & 6.63 & 7.04 & 4.00 \\
16 & 5.51 & 7.86 & 5.42 & 7.09 & 8.60 \\
24 & 5.26 & 8.78 & 5.26 & 7.48 & 9.20 \\
48 & 4.48 & 9.18 & 4.84 & 7.91 & 8.41 \\
72 & 4.33 & 9.30 & 4.46 & 9.18 & 5.90 \\
\hline
\end{tabular}

Tabla 2. Valores de pH y recuentos de Cronobacter sakazakii y probióticos en los preparados reconstituidos para lactantes y almacenadas a $40{ }^{\circ} \mathrm{C}$

\begin{tabular}{|c|c|c|c|c|c|}
\hline \multirow{2}{*}{$\begin{array}{l}\text { Tiempo de } \\
\text { incubación } \\
\text { (horas) }\end{array}$} & \multicolumn{2}{|c|}{ Fórmula sin probióticos } & \multicolumn{3}{|c|}{ Fórmula con probióticos } \\
\hline & $\mathrm{pH}$ & $\begin{array}{l}\text { C. sakazakii(log } \\
\mathrm{UFC} / \mathrm{mL})\end{array}$ & $\mathrm{pH}$ & $\begin{array}{l}\text { Probióticos }(\log \\
\text { UFC } / \mathrm{mL})\end{array}$ & $\begin{array}{l}\text { C. sakazakii }(\log \\
\text { UFC/mL) }\end{array}$ \\
\hline 0 & 6.81 & 2.15 & 6.67 & 7.00 & 2.62 \\
\hline 4 & 6.83 & 4.00 & 6.71 & 7.20 & 4.00 \\
\hline 16 & 4.98 & 8.78 & 4.74 & 7.56 & 7.69 \\
\hline 24 & 4.50 & 7.86 & 4.37 & 7.71 & 7.41 \\
\hline 48 & 4.35 & 6.00 & 3.88 & 8.13 & nd \\
\hline 72 & 4.29 & nd & 3.84 & 8.79 & nd \\
\hline
\end{tabular}

nd: no detectado 
menor que en la muestra sin probióticos. Esto supone una disminución del $36.5 \%$ en los recuentos de C. sakazakii, sugiriendo el posible efecto antimicrobiano de los probióticos frente a C. sakazakii a $30^{\circ} \mathrm{C}$ a partir de las 48 horas. Los recuentos de $C$. sakazakii no se recuperaron en las muestras con probióticos a las 48 horas de incubación a $40{ }^{\circ} \mathrm{C}$, mientras que si se aisló la bacteria en ausencia de las bacterias lácticas. Por tanto, el efecto inhibitorio de los probióticos frente $C$. sakazakii se ve potenciado por la temperatura de incubación. Tal y como se observa en la Tabla 1 y 2, los valores de $\mathrm{pH}$ de los preparados de continuación disminuyeron significativamente $(\mathrm{p}<0,05)$ durante la incubación, con independencia de las condiciones de estudio.
La Tabla 3 y 4 muestran los valores de $\mathrm{pH}$ y recuentos de Salmonella y probióticos obtenidos a partir de las muestras reconstituidas almacenadas a 30 y $40{ }^{\circ} \mathrm{C}$ respectivamente. Los recuentos bacterianos de Salmonella no disminuyeron durante las 72 horas de incubación, independientemente de las condiciones de estudio. Al igual que C. sakazakii, los valores de $\mathrm{pH}$ de los preparados para lactantes disminuyeron significativamente $(p<0,05)$ durante la incubación a 30 y $40{ }^{\circ} \mathrm{C}$.

La Figura 2 ilustra la evolución del crecimiento de la mezcla de probióticos presente en las muestras reconstituidas. Durante las 72 horas de incubación, se observa un aumento constante de la población de las bacterias lácticas

\section{Tabla 3. Valores de pH y recuentos Salmonella enterica y probióticos de los preparados re- constituidos para lactantes y almacenadas a $30{ }^{\circ} \mathrm{C}$}

\begin{tabular}{lccccc}
\hline $\begin{array}{l}\text { Tiempo de } \\
\text { incubación } \\
\text { (horas) }\end{array}$ & Preparados sin probióticos & \multicolumn{3}{l}{ Preparados con probióticos } & \\
\cline { 2 - 6 } & $\mathrm{pH}$ & $\begin{array}{l}\text { Salmonella } \\
(\log \text { UFC/mL) }\end{array}$ & $\mathrm{pH}$ & $\begin{array}{l}\text { Probióticos } \\
(\log \text { UFC/mL) }\end{array}$ & $\begin{array}{c}\text { Salmonella } \\
(\log \text { UFC/mL) }\end{array}$ \\
\hline 0 & 6.82 & 1.65 & 6.62 & 7.00 & 2.08 \\
4 & 6.79 & 2.00 & 6.67 & 7.08 & 5.00 \\
16 & 5.54 & 8.86 & 5.45 & 7.12 & 8.45 \\
24 & 5.08 & 8.78 & 5.14 & 7.11 & 8.60 \\
48 & 4.48 & 10.30 & 4.52 & 7.59 & 8.00 \\
72 & 4.29 & 6.04 & 4.39 & 8.92 & 8.65 \\
\hline
\end{tabular}

Tabla 4. Valores de pH y recuentos Salmonella enterica y probióticos de los preparados reconstituidos para lactantes y almacenados a $40{ }^{\circ} \mathrm{C}$

\begin{tabular}{lccccc}
\hline $\begin{array}{l}\text { Tiempo de } \\
\text { incubación } \\
\text { (horas) }\end{array}$ & \multicolumn{4}{l}{ Preparados sin probióticos } & \multicolumn{3}{l}{ Preparados con probióticos } & \\
\cline { 2 - 6 } 0 & $\mathrm{pH}$ & $\begin{array}{l}\text { Salmonella } \\
(\log \text { UFC/mL) }\end{array}$ & $\mathrm{pH}$ & $\begin{array}{l}\text { Probióticos } \\
(\log \text { UFC/mL) }\end{array}$ & $\begin{array}{c}\text { Salmonella } \\
(\log \text { UFC/mL) }\end{array}$ \\
\cline { 2 - 6 } 0 & 6.80 & 2.04 & 6.65 & 7.00 & 1.90 \\
4 & 6.78 & 2.00 & 6.58 & 7.26 & 2.70 \\
16 & 5.16 & 8.39 & 5.06 & 7.46 & 8.41 \\
24 & 4.71 & 8.60 & 4.52 & 7.49 & 8.13 \\
48 & 4.49 & 7.30 & 3.71 & 8.20 & 8.91 \\
72 & 4.35 & nd & 3.70 & 9.94 & nd \\
\hline
\end{tabular}

nd: no detectado 


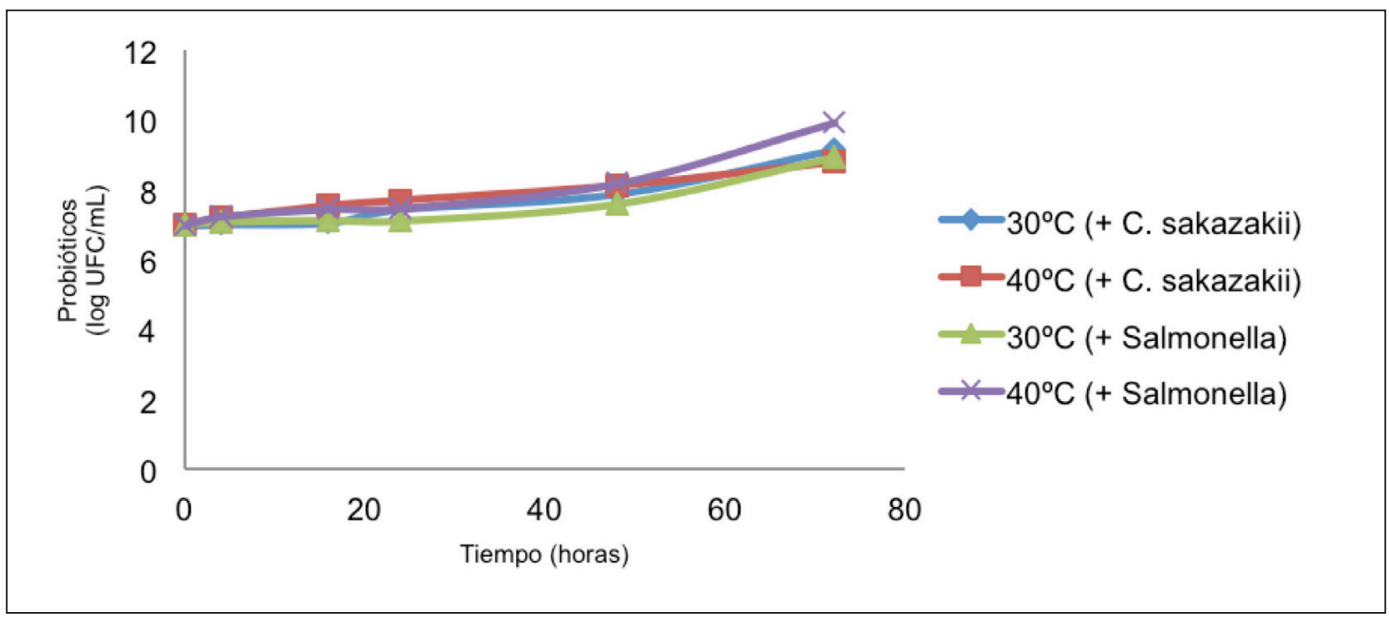

Figura 2. Evolución del crecimiento de la mezcla de probióticos presente en los preparados para lactantes reconstituidas bajo las condiciones de estudio

del preparado, con independencia de la temperatura de incubación y de las variaciones de $\mathrm{pH}$.

\section{DISCUSIÓN}

Nuestros hallazgos sugieren que la mezcla de probióticos presente en el preparado para lactantes fue capaz de inhibir el crecimiento de C. sakazakii, efecto que se vio incrementado por la temperatura de incubación. Al igual que en este experimento, Shaker et al. (2008) informaron de la inhibición de $C$. sakazakii durante el procesamiento y almacenamiento de yogur natural con Lactobacillus bulgaricus y Streptococcus thermophilus. La competencia por los nutrientes en la matriz alimentaria podría contribuir a la disminución de la población de C. Sakazakii. Sin embargo, los probióticos continuaron creciendo a lo largo del período de incubación, por lo que el agotamiento de nutrientes se descarta como mecanismo de inhibición del crecimiento de $C$. sakazakii. En nuestro estudio los valores de $\mathrm{pH}$ más bajos se observaron a las 48 y $72 \mathrm{~h}$, coincidiendo con los recuentos más bajos de $C$. sakazakii, sugiriendo que la acidificación del medio por la producción ácidos orgánicos u otros metabolitos -peróxido de hidrógeno, dióxido de carbono y diacetilo-, derivados del crecimiento de las bacterias acidolácticas, podría desempeñar un papel clave en la inhibición de $C$. sakazakii.

Por otra parte, la síntesis de péptidos con actividad antimicrobiana -nisina, lactobrevina, acidofilina- por parte de los probióticos puede inhibir el crecimiento de una amplia gama de patógenos transmitidos por los alimentos, dependiendo de su especificidad (Mishra and Lambert, 1996; O'Connor et al., 2020). L. acidophilus DPC 6026 produce sustancias proteicas derivadas de la caseína capaces de inhibir el crecimiento de bacterias como E. coli y $E$. sakazakii (Hayes et al., 2006, 2009). Igualmente, otros autores han demostrado el potencial inhibidor de diversas cepas de BAL sobre el crecimientos de C. sakazakii (Kim et al., 2018; Campana et al., 2019; Jamwal et al., 2019), aunque otros estudios han demostrado resultados contradictorios (Al-Holy et al., 2009). La actividad antibacteriana del probiótico $L$. acidophilus ha sido examinada y los resultados 
han demostrado que este probiótico no ha sido efectivo para inhibir C. sakazakii en preparados para lactantes en polvo o reconstituidas. Del mismo modo, otro estudio mostró que el probiótico Bifidobacterium breve no redujo la concentración de $C$. sakazakii en fórmulas infantiles reconstituidas a más de $30{ }^{\circ} \mathrm{C}$, incluso fue capaz de estimular el crecimiento del patógeno tras 2 horas de incubación a $37{ }^{\circ} \mathrm{C}$ (Osaili et al., 2008). Estos hallazgos concuerdan con los resultados de nuestro estudio en los que la presencia de probióticos en los preparados reconstituidos para lactantes no tiene efecto inhibitorio sobre el patógeno Salmonella en las condiciones probadas. Por tanto, las posibles bacteriocinas secretadas por los probióticos durante su crecimiento podrían explicar el efecto inhibitorio sobre el crecimiento de $C$. sakazakii pero no frente a Salmonella. Sin embargo, la posibilidad de que, frente a $\mathrm{pH}$ más ácidos, $C$. sakazakii sea más sensible que Salmonellla no debería descartarse, requiriéndose más estudios para corroborar estas hipótesis.

Los probióticos mantuvieron su viabilidad en las muestras reconstituidas durante las 72 horas de estudio. Este dato es de interés ya que proporciona información sobre la estabilidad de los componentes funcionales de los preparados para lactantes durante su vida útil. Esta supervivencia de los probióticos puede ser muy útil frente a la posible contaminación con microorganismos patógenos, aunque la actividad antimicrobiana del probiótico -o acción simbiótica de pre y probióticos- se espera que se produzca principalmente en el tracto gastrointestinal.

Las condiciones utilizadas en nuestro estudio son improbables siempre y cuando se apliquen las recomendaciones en la reconstitución de preparados para lactantes (EFSA, 2004; WHO/FAO, 2007)PIF can become contaminated with harmful bacteria, such as Enterobacter sakazakii and Salmonella enterica. This is because, using current manufacturing technology, it is not feasible to produce sterile PIF. During the preparation of PIF, inappropriate handling practices can exacerbate the problem. Recognizing the need to address such hazards in PIF, Codex Alimentarius decided to revise the Recommended International Code of Hygienic Practice for Foods for Infants and Children. In doing so it requested specific scientific advice from the Food and Agriculture Organization of the United Nations (FAO. El enfoque experimental utilizado en este estudio sirve para crear un hipotético ambiente en el cual se puede revelar el posible efecto antimicrobiano de los probióticos frente a C. sakazakii y Salmonella enterica.

\section{CONCLUSIONES}

Este estudio demuestra la estabilidad de los probióticos de los preparados para lactantes suplementados durante su vida útil. La presencia de probióticos no mostró ninguna actividad inhibitoria frente a Salmonella, mientras que la actividad inhibitoria contra $C$. sakazakii puede deberse a la producción de bacteriocinas específicas contra este enteropatógeno que actúan en función de la temperatura.

\section{AGRADECIMIENTOS}

Este trabajo fue apoyado por la Universidad de Murcia y el Campus Regional de Excelencia Internacional Campus Mare Nostrum.

\section{REFERENCIAS}

Al-Holy, M.A., Lin, M., Abu-Ghoush, M.M., Al-Qadiri, H.M., Rasco, B.A., 2009. Thermal Resistance, Survival and Inactivation of Enterobacter Sakazakii (Cronobacter spp.) in Powdered and Reconstituted Infant Formula. J. Food Saf. 29, 287-301. https://doi. org/10.1111/j.1745-4565.2009.00157.x

Al-Nabulsi, A.A., Osaili, T.M., Elabedeen, N.A.Z., Jaradat, Z.W., Shaker, R.R., Kheirallah, K.A., Tarazi, Y.H., Holley, R.A., 2011. Impact of environmental stress desiccation, 
acidity, alkalinity, heat or cold on antibiotic susceptibility of Cronobacter sakazakii. Int. J. Food Microbiol. 146, 137-143. https:// doi.org/10.1016/j.ijfoodmicro.2011.02.013

Bai, Y., Yu, H., Du, G., Fei, S., Shi, C., 2019. Survival and Environmental Stress Resistance of Cronobacter sakazakii Exposed to Vacuum or Air Packaging and Stored at Different Temperatures. Drontiers in Microbiology, 10, 303

Braegger, C., Chmielewska, A., Decsi, T., Kolacek, S., Mihatsch, W., Moreno, L., Pieścik, M., Puntis, J., Shamir, R., Szajewska, H., Turck, D., Van Goudoever, J., 2011. Supplementation of infant formula with probiotics and/or prebiotics: A systematic review and comment by the ESPGHAN committee on nutrition. J. Pediatr. Gastroenterol. Nutr. 52, 238-250. https://doi.org/10.1097/ MPG.0b013e3181fb9e80

Campana, R., Federici, S., Ciandrini, E., Manti, A., Baffone, W. 2019. Lactobacillus spp. inhibit the growth of Cronobacter sakazakii ATCC 29544 by altering its membrane integrity. J Food Sci Technol 56, 39623967.

Comisión Europea, 2016. REGLAMENTO DELEGADO (UE) 2016/127 DE LA COMISIÓN de 25 de septiembre de 2015. D. Of. la Unión Eur. 1-25. https://doi.org/10.1166/ apm.2014.1068

Dancer, G.I., 2009. Enterobacter sakazakii (Cronobacter spp.) in powdered follow-up formulae. Lett. Appl. Microbiol. 10 , 173. https://doi.org/10.1111/j.1472765X.2009.02601.X

Drudy, D., Mullane, N.R., Quinn, T., Wall, P.G., Fanning, S., 2006. Enterobacter sakazakii: An Emerging Pathogen in Powdered Infant Formula. Clin. Infect. Dis. 42, 996-1002. https://doi.org/10.1086/501019

EFSA, 2004. Opinion of the Scientific Panel on Biological Hazards on the request from the Commission related to the microbiological risks in infant formulae and Adopted on 9 th
September 2004. EFSA J. 113, 1-35. https:// doi.org/10.1016/0368-2048(73)80049-0

FAO/WHO, 2006. Enterobacter sakazakii and Salmonella in powdered infant formula: meeting report. Microbiological Risk Assessment.

Ghassem, M., Babji, A.S., Forsythe, S.J., Norrakiah, A.S., 2011. Growth and survival of Cronobacter species as measured by media performance. Int. Food Res. J. 18, 367-372.

Hayes, M., Barrett, E., Ross, R.P., Fitzgerald, G.F., Hill, C., Stanton, C., 2009. Evaluation of an Antimicrobial Ingredient Prepared from a Lactobacillus acidophilus Casein Fermentate against Enterobacter sakazakii. J. Food Prot. 72, 340-346. https://doi. org/10.1182/blood.V100.1.22

Hayes, M., Ross, R.P., Fitzgerald, G.F., Hill, C., Stanton, C., 2006. Casein-Derived Antimicrobial Peptides Generated by Lactobacillus acidophilus DPC6026. Appl. Environ. Microbiol. 72, 2260-2264. https://doi. org/10.1097/00019501-199501000-00002

Hurrell, E., Kucerova, E., Loughlin, M., Caubilla-Barron, J., Forsythe, S.J., 2009. Biofilm formation on enteral feeding tubes by Cronobacter sakazakii, Salmonella serovars and other Enterobacteriaceae. Int. J. Food Microbiol. 136, 227-231. https://doi. org/10.1016/j.ijfoodmicro.2009.08.007

Iversen, C., Lane, M., Forsythe, S.J., 2004. The growth profile, thermotolerance and biofilm formation of Enterobacter sakazakii grown in infant formula milk. Lett. Appl. Microbiol. 38, 378-382. https://doi.org/10.1111/ j.1472-765X.2004.01507.x

Jamwal, A., Sharma, K., Chauhan, R., Bansal, S., Goel, G., 2019. Evaluation of commercial probiotic lactic cultures against biofilm formation by Cronobacter sakazakii. Intest Res.17(2), 192-201

Kandhai, M.C., Reij, M.W., Grognou, C., Van Schothorst, M., Gorris, L.G.M., Zwietering, M.H., 2006. Effects of preculturing conditions on lag time and specific growth rate 
of Enterobacter sakazakii in reconstituted powdered infant formula. Appl. Environ. Microbiol. 72, 2721-2729. https://doi. org/10.1128/AEM.72.4.2721-2729.2006

Kim, D.H., Jeong, D., Song, K.Y., Kang, I.B., Kim, H., Seo, K.H., 2018. Culture supernatant produced by Lactobacillus kefiri from kefir inhibits the growth of Cronobacter sakazakii. J Dairy Res, 85, 98-103.

Kingsbury, J.M., Thom, K., Soboleva, T., 2019. Effect of Storage Temperature on the Survival of New Zealand Egg-Associated Salmonella Isolates in and on Eggs. J Food Prot. 82 (12), 2161-2168.

Kopp-Hoolihan, L., 2001. Prophylactic and therapeutic uses of probiotics: A review. J. Am. Diet. Assoc. https://doi.org/10.1016/ S0002-8223(01)00060-8

Lenati, R.F., O'Connor, D.L., Hébert, K.C., Farber, J.M., Pagotto, F.J., 2008. Growth and survival of Enterobacter sakazakii in human breast milk with and without fortifiers as compared to powdered infant formula. Int. J. Food Microbiol. 122, 171-179. https:// doi.org/10.1016/j.ijfoodmicro.2007.11.084

Markowiak, P., Ślizewska, K., 2017. Effects of probiotics, prebiotics, and synbiotics on human health. Nutrients 9. https://doi. org/10.3390/nu9091021

Mishra, C., Lambert, J., 1996. Production of anti-microbial substances by probiotics. Asia Pacific J. Clin. Nat. 5, 20-24.

O'Connor, P.M., Kuniyoshi, T.M., Oliveira, R.P., Hill, C., Ross, R.P., Cotter, P.D., 2020. Antimicrobials for food and feed; a bacteriocin perspective. Current Opinion in Biotechnology, 61:160-167.
Osaili, T.M., Shaker, R.R., Ayyash, M.M., Holley, R.A., 2008. Effect of Bifidobacterium breve on the survival and growth of Enterobacter sakazakii in rehydrated infant milk formula. J. Food Prot. 28, 36-46.

Park, J., Soo-Hwan, K., 2007. Thermal Resistance and Inactivation of Rehydration of Powdered Infant Formula. J. Microbiol. Biotechnol. 17, 364-368.

Salimiyan, R.K., Ghazvini K., Farsiani H., 2019. Clinical and pathogenesis overview of Enterobacter infections. Rev Clin Med. 6(4), 146-154.

Shaker, R.S., Osaili, T.M., Ayyash, M., 2008. Effect of thermophilic lactic acid bacteria on the fate of Enterobacter sakazakii during processing and storage of plain yogurt. J. Food Saf. 170-182. https://doi.org/10.3109 /08958378.2012.757400

Simón, M. De, Sabaté, S., Cristina Osanz, A., Bartolomé, R., Dolores Ferrer, M., 2010. Investigación de un caso de infeccin neonatal por Enterobacter sakazakii asociada a un preparado en polvo para lactantes. Enferm. Infecc. Microbiol. Clin. 28, 713-715. https://doi.org/10.1016/j.eimc.2010.04.009

Usera, M.A., Echeita, A., Aladuena, A., Blanco, M.C., Reymundo, R., Prieto, M.I., Tello, O., Cano, R., Herrera, D., Martinez-Navarro, F., 1996. Interregional foodborne salmonellosis outbreak due to powdered infant formula contaminated with lactose-fermenting Salmonella Virchow. Eur. J. Epidemiol. 12, 377-381. https://doi.org/10.1007/ BF00145301

WHO/FAO, 2007. Storage and handling of powdered infant formula: guidelines. 
